\title{
Using Biological Variation Data for Reference Change Values
}

\section{in Clinical Laboratories}

\section{Ozlem Goruroglu Ozturk*}

Faculty of Medicine, Department of Clinical Biochemistry and Cukurova University, Balcali Hospital, Central Laboratory, Adana, Turkey

Biological Variation (BV) of quantities examined in laboratory medicine can be described as of three types, namely; variation over the span of life, predictable cyclical variation that can be daily, monthly or seasonal in nature, and random variation [1]. Random variations of analytes which consist of random fluctuation around the setting point of each individual is known as the intra-individual biological variation. Additionally, each person's setting point may be different from another's, and the overall variation resulting from this difference is known as inter-individual biological variation. In mathematical terms, these are usually expressed as Coefficients Of Variation (CV) and termed as $\mathrm{CVw}$ for intra-individual $\mathrm{BV}$ and $\mathrm{CVg}$ for inter-individual $\mathrm{BV}[2]$.

In laboratory medicine, it is essential to take the BV concept into consideration to provide reliable results. Thus, clinical laboratories should minimize all the sources of variations related laboratory processes, estimate the components of $\mathrm{BV}$ and appropriately manage them during the entire process leading to the laboratory report. A comprehensive database constituted from BV data of nearly 320 analytes which is updated every 2 years serves as a useful reference for many clinical laboratories [3,4]. Nonetheless, there are still hundreds of constituents for which BV has not yet been estimated. Future work should focus on these specific tests.

Clinicians use several approaches in the interpretation of routine laboratory tests of the patients. These include comparison with predetermined cut-off values or reference values, or a comparison between two sequential results for a specific analyte [5]. Comparison between two sequential results is not as straightforward as it seems. It should be remembered that each result has its own inherent random variation associated with laboratory activity (analytical variation, CVa) and Biological Variation (BV) [6]

It has become clear that conventional population based reference values do have serious intrinsic problems and thus, there is a need for revisiting practical applications of conventional population based reference values for getting more useful laboratory data [7]. A major problem with reference values is that biological quantities are not constants that can be measured once in one reference sample group to provide reference values that are applicable in all situations. Knowledge of the underlying BV of analytes examined in laboratories is vital to understanding the proper generation and application of traditional population based reference values. Using the BV data is the best way to detect changes in a patient's health status through a comparison between serial analytical results rather than comparison with population based reference values. This is because of the marked individuality of the majority of analytes. Hence, values obtained in consecutive analyses of samples from a patient may fall within the reference range, but show a considerable difference. When the difference exceeds a certain value, known as the Reference Change Value (RCV), a change in the patient's condition is indicated $[7,8]$.

The usefulness of reference intervals has been addressed by the concept of biological individuality also referred to as the "index of individuality" (II) which is a ratio of CVw to CVg. When the index is lower than 1 , which is usual for the majority of analytes compiled up-to-date, two consecutive results from a subject may be outside the RCV but well within the population-based reference interval. As a new concept, comparison of the result of a single test with the populationbased reference interval is a satisfactory practice only for analytes with the index of individuality higher than $1[8,9]$.

The RCV, defined as the critical difference that must be exceeded between two sequential results for a significant (or true) change to occur incorporates the total variation associated with both results and is demonstrated by an equation $\left[\mathrm{RCV}=2^{1 / 2} * \mathrm{Z}^{\star}\left(\mathrm{CVa}^{2}+\mathrm{CVw}^{2}\right)^{1 / 2}\right]$. This equation is based on the the random variations associated with a result follows a Gaussian distribution [2]. Important factors of the equation to consider are the $\mathrm{Z}$-score (i.e. the desired level of statistical significance; $Z=1.96$ for $95 \%$ significance when evaluating a bidirectional change), the analytical variation (CVa) (usually, $5 \%$ for automated tests) $[10]$ and the intra-individual $\mathrm{BV}(\mathrm{CVw})$ which is often the largest contributor to the variation [7]. By this RCV concept clinicians could be alerted to these possible intrinsic variations that may influence the magnitude of the difference between serial results [11].

Collaboration between clinical laboratories and clinicians is becoming more necessary as the complexity of the diagnostic processes is growing up. It has become more common to use RCVs estimated from BV data of healthy subjects to detect significant changes in the status of patient. Additionally it has been proposed previously that RCV can be used for delta checking and autoverification: Laboratory information managment systems (LIMS) can be adapted to do this, as has been demonstrated [12]. Using of RCV model could be valuable for follow up results and does not include extra cost for the clinical laboratory. It only requires a LIMS in which a reliable algorithm can be programmed to relate the diagnosis, patient's prior results and the present values. When the assigned percentages (RCVs) for analytes exceeded simultaneously, a warning message should be created by the LIMS to inform the clinician that the clinical status of patients may be undergoing towards an unfavorable change. Laboratories should better use this model as a routine aplication for improving the interpretation of laboratory reports.

\section{References}

1. Fraser CG, Harris EK (1989) Generation and application of data on biological variation in clinical chemistry. Crit Rev Clin Lab Sci 27: 409-437.

*Corresponding author: Ozlem Goruroglu Ozturk, Faculty of Medicine, Department of Clinical Biochemistry and Cukurova University, Balcali Hospital, Central Laboratory, Adana, Turkey, E-mail: ozlem_goruroglu@yahoo.com

Received May 21, 2012; Accepted May 23, 2012; Published May 25, 2012

Citation: Ozturk OG (2012) Using Biological Variation Data for Reference Change Values in Clinical Laboratories. Biochem Anal Biochem 1:e106. doi:10.4172/21611009.1000e106

Copyright: (c) 2012 Ozturk OG. This is an open-access article distributed under the terms of the Creative Commons Attribution License, which permits unrestricted use, distribution, and reproduction in any medium, provided the original author and source are credited. 
Citation: Ozturk OG (2012) Using Biological Variation Data for Reference Change Values in Clinical Laboratories. Biochem Anal Biochem 1:e106. doi:10.4172/2161-1009.1000e106

Page 2 of 2

2. Fraser CG (2001) Biological Variation: From Principles to Practice. American Association for Clinical Chemistry, Washington DC

3. Ricos C, Alvarez V, Cava F, Garcia-Lario JV, Hernandez A, et al. (1999) Current databases on biological variation: pros, cons and progress. Scand J Clin Lab Invest 59: 491-500.

4. Desirable Biological Variation Database specifications.

5. Ricos C, Cava F, Garcia-Lario JV, Hernández A, Iglesias N, et al. (2004) The reference change value: a proposal to interpret laboratory reports in serial testing based on biological variation. Scand J Clin Lab Invest 64: 175-184.

6. Omar F, van der Watt GF, Pillay TS (2008) Reference change values: how useful are they? J Clin Pathol 61: 426-427.

7. Fraser CG (2004) Inherent biological variation and reference values. Clin Chem Lab Med 42: 758-764.
8. Ricos C, Perich C, Minchinela J, Alvarez V, Simon S, et al. (2009) Application of biological variation - a review. Biochemia Medica 19: 250-259.

9. Petersen PH, Fraser CG, Sandberg S, Goldschmidt H (1999) The index of individuality is often a misinterpreted quantity characteristic. Clin Chem Lab Med 37: 655-661.

10. Waugh JM, Collier CP, Day AG, Waugh M, Raymond MJ (2002) Proficiency testing performance: a case study with modeling. Clin Biochem 35: 447-453.

11. Smellie WS (2008) What is a significant difference between sequentia laboratory results? J Clin Pathol 61: 419-425.

12. Fraser CG, Stevenson HP, Kennedy IMG (2002) Biological variation data are necessary prerequisites for objective autoverification of clinical laboratory data Accred Qual Assur 7: 455-460. 